\title{
Perturbed bifurcations in the BCS gap equation
}

\author{
Spathis, P. N.; Sørensen, Mads Peter; Lazarides, Nickos
}

Published in:

Physical Review B

Link to article, DOI:

10.1103/PhysRevB.45.7360

Publication date:

1992

Document Version

Publisher's PDF, also known as Version of record

Link back to DTU Orbit

Citation (APA):

Spathis, P. N., Sørensen, M. P., \& Lazarides, N. (1992). Perturbed bifurcations in the BCS gap equation. Physical Review B, 45(13), 7360-7367. https://doi.org/10.1103/PhysRevB.45.7360

\section{General rights}

Copyright and moral rights for the publications made accessible in the public portal are retained by the authors and/or other copyright owners and it is a condition of accessing publications that users recognise and abide by the legal requirements associated with these rights.

- Users may download and print one copy of any publication from the public portal for the purpose of private study or research.

- You may not further distribute the material or use it for any profit-making activity or commercial gain

- You may freely distribute the URL identifying the publication in the public portal

If you believe that this document breaches copyright please contact us providing details, and we will remove access to the work immediately and investigate your claim. 


\title{
Perturbed bifurcations in the BCS gap equation
}

\author{
P. N. Spathis and M. P. Soerensen \\ Laboratory of Applied Mathematical Physics, The Technical University of Denmark, Building 303, DK-2800 Lyngby, Denmark \\ N. Lazarides \\ Research Center of Crete, P.O. Box 1529, 71110 Heraklion, Crete, Greece \\ (Received 11 June 1991; revised manuscript received 3 October 1991)
}

\begin{abstract}
The anisotropic BCS gap equation is applied to a tight-binding model of layered high-temperature superconductors. The possible solutions have $s$-, $d$-, and mixed $s$ - and $d$-wave symmetries using nearestneighbor intralayer singlet pairing interaction of the same strength in the $x$ and $y$ directions. The transitions from $d$ - or $s$ - to mixed $s$ - and $d$-wave solutions result from pitchfork bifurcations. In the case of slightly different pairing strength in the $x$ and $y$ directions, perturbed pitchfork bifurcations emerge, leading to a dramatic change in the physical properties of the superconducting state.
\end{abstract}

\section{INTRODUCTION}

Recently, the classical BCS strategy has been employed for the study of high-temperature superconductors by a number of authors. ${ }^{1-7}$ In these studies the BCS effective Hamiltonian has been applied with pairing mechanisms different from the usual phonon-mediated interaction found in the classical low-temperature superconductors. Many proposals for the origin of the electron-electron or hole-hole interaction have been put forward. ${ }^{1,3,8-15}$ To name a few we mention coupling to local spin configurations on the $\mathrm{Cu}$ sites, ${ }^{1}$ charge-transfer excitations, ${ }^{3}$ electron-electron attraction originating from coupling between electrons and excitons or plasmons, a purely electronic mechanism, ${ }^{8,9,10}$ spin-polaron pairing, ${ }^{11,12}$ image-charge-mediated pairing, ${ }^{13}$ a bisoliton mechanism, ${ }^{14}$ combined spinon and holon pairing, ${ }^{15}$ etc. Also, for non-phonon-mediated pairing, the BCS strategy can be applied. ${ }^{1,3-11,15}$ The numerous investigations ${ }^{1-7}$ based on the BCS Hamiltonian in the study of high- $T_{c}$ superconductors justifies a more detailed study of the nonlinear properties of the BCS gap equation. ${ }^{16}$

We shall adopt the BCS method to a particular simple tight-binding model of layered high- $T_{c}$ superconductors, assuming a single-band structure and nearest-neighbor unretarded hole-hole interaction within the layers of $\mathrm{CuO}_{2} \cdot{ }^{7}$ The pairing interaction is treated phenomenologically without reference to a specific microscopic mechanism. The gap is parametrized into two gap variables describing gap anisotropy in the $x$ and $y$ directions, respectively. In this case the BCS gap equation reduces to a two-dimensional nonlinear algebraic equation for the gap variables which depend on parameters such as the absolute temperature, chemical potential or filling, interaction strength, and band variables.

Here we shall study various bifurcation phenomena in the above two variable-gap equations and their implications on measurable quantities such as the electronic specific heat and spin susceptibility. The transition from the normal to the superconducting state is due to a pitchfork bifurcation in the gap equation. In Ref. 16 further transitions resulting from pitchfork bifurcations have been found, converting solutions with $s$ - or $d$-wave symmetry into solutions with mixed $s$ - and $d$-wave symmetries. Here we shall explore perturbations of these latter pitchfork bifurcations following from slightly nonsymmetrical pairing interactions in the $x y$ plane; i.e., the pairing interactions in the $x$ direction deviate from the pairing strength in the $y$ direction. This asymmetry in the pairing strength is expected because the lattice constants in the $\mathrm{CuO}_{2}$ planes are not equal, ${ }^{17}$ leading to a small difference in the pairing strength in the $x$ and $y$ directions.

In Sec. II we present the BCS model for the asymmetric pairing interaction. Section III discuss pitchfork bifurcations in the BCS gap equation. Section IV contains numerical results in the case of the asymmetric pairing interaction compared with the symmetric case. The dramatic consequence of this symmetry breaking is demonstrated by calculating the electronic specific heat and spin susceptibility. Finally, Sec. V concludes our study.

\section{MODEL DESCRIPTION}

Assuming tight-binding Fermi-liquid states, subject to an unretarded pairing interaction, we take as our starting point the Hamiltonian $7,16,18$

$$
H=\sum_{i, j, \sigma} t_{i, j} a_{i, \sigma}^{\dagger} a_{j, \sigma}-\sum_{i, j} g_{i, j} a_{i, \uparrow}^{\dagger} a_{j, \downarrow}^{\dagger} a_{j, \downarrow} a_{i, \uparrow}
$$

The operators $a_{i, \sigma}^{\dagger}\left(a_{i, \sigma}\right)$ creates (annihilates) quasiparticle holes with spin $\sigma$ at site $i$. The $t_{i, j}$ term describes the hopping of quasiparticles within and between layers, and $g_{i, j}$ denotes the pairing strength between quasiparticles at sites $i$ and $j$. Denoting the overlap integrals between nearest neighbors within planes by $t_{i, j}=t_{1}=t_{2}=-A / 2$, those between next-nearest neighbors by $t_{i j}=t_{3}=A B$, and, finally, those between planes by $t_{i, j}=t_{4}=-A C$, the quasiparticle dispersion reads 


$$
\begin{aligned}
\varepsilon_{\mathrm{k}}=A\{ & -2\left[\cos \left(k_{x} a_{x}\right)+\cos \left(k_{y} a_{y}\right)\right] \\
& \left.+4 B \cos \left(k_{x} a_{x}\right) \cos \left(k_{y} a_{y}\right)-2 C \cos \left(k_{z} a_{z}\right)-\mu\right\},
\end{aligned}
$$

where $\mu$ is the chemical potential. The model structure and hopping terms are depicted in Fig. 1(a), and Fig. 1(b) shows the pairing strength between neighboring sites. In Eq. (2) we assume a unit cell with lattice constants $a_{x}, a_{y}, a_{z}$ and $k_{\alpha} a_{\alpha}=n_{\alpha} \pi / N_{\alpha},-N_{\alpha}<n_{a} \leq N_{a}, \alpha=x, y, z$. The individual lattice sites should be thought of as hybrid states of $\mathrm{Cu} d$ states and $\mathrm{O} p \sigma$ states. An example could be the oxygen and copper diamond states described in Ref. 19, considering only one band. Representative values of the lattice constants are $a_{x}=3.82 \AA, a_{y}=3.89$ $\AA$, and $a_{z}=11.68 \AA$. These values are for the crystal structure of $\mathrm{YBa}_{2} \mathrm{Cu}_{3} \mathrm{O}_{7}{ }^{17}$ As $a_{x}$ is slightly different from $a_{y}$, we may expect that the hopping probability and pairing interaction in the $x$ and $y$ directions also differ slightly. In the dispersion relation (2), we have assumed identical hopping probability in the two directions within the planes $\left(t_{1}=t_{2}\right)$, but the pairing interactions in the $x$ and $y$ directions we shall assume asymmetric and compare the results with the symmetric case. The parameters $A, B$, and $C$ in the dispersion relation (2) can be fitted to band-structure calculations ${ }^{17,19,20}$ or to angle-resolved

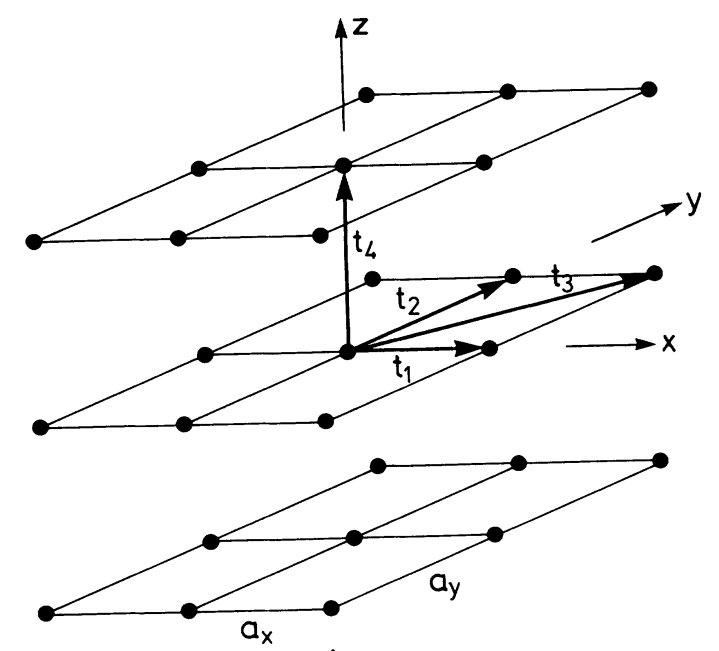

(a)

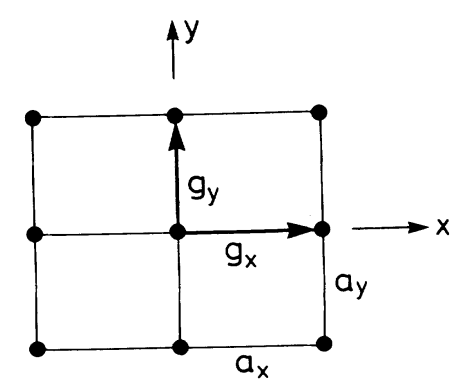

(b)

FIG. 1. (a) Hopping probabilities between nearest and nextnearest neighbors and between planes. (b) The pairing interaction strengths between sites within the $x y$ plane. photoemission data. ${ }^{21,22}$ Typical bandwidths found from band-structure calculations vary between 2 and $5 \mathrm{eV}$, whereas the photoemission experiments state values of magnitudes $0.2-0.5 \mathrm{eV}$ for widths of bands crossing the Fermi surface. The bandwidth of the spectrum in Eq. (2) is $A(8+4 C)$ and, by knowing $C$ together with a typical experimental value for the bandwidth, then gives $A$. In the following we shall choose a value of $A$ which is in accord with the photoemission experiments, and the values of $B$ and $C$ are taken from Ref. 7; i.e., we choose

$$
A=0.05 \mathrm{eV}, B=0.45, C=0.1 \text {. }
$$

Note that the above values of $A$ and $C$ correspond to a bandwidth of $0.42 \mathrm{eV}$.

Restricting the pairing interaction to nearest neighbors within the planes [see Fig. 1(b)], the pairing potential $V$ in Fourier space reads ${ }^{7}$

$V\left(\mathbf{k}, \mathbf{k}^{\prime}\right)=2 g_{x} \cos \left[\left(k_{x}-k_{x}^{\prime}\right) a_{x}\right]+2 g_{y} \cos \left[\left(k_{y}-k_{y}^{\prime}\right) a_{y}\right]$,

where $g_{x}$ and $g_{y}$ denote the strength of the hole-hole interaction in the $x$ and $y$ directions. Adopting the BCS strategy, the Gibbs free energy evaluated for the BCS trial wave function is given $b^{23}$

$$
\begin{aligned}
G\left(\Delta_{\mathbf{k}}\right)= & \frac{1}{N} \sum_{\mathbf{k}}\left[\varepsilon_{\mathbf{k}}-E_{\mathbf{k}}+2 \Delta_{\mathbf{k}} F_{\mathbf{k}}^{*}-\frac{2}{\beta} \ln \left[1+\exp \left(-\beta E_{\mathbf{k}}\right)\right]\right] \\
& -\frac{1}{N^{2}} \sum_{\mathbf{k}} \sum_{\mathbf{k}^{\prime}} V\left(\mathbf{k}, \mathbf{k}^{\prime}\right) F_{\mathbf{k}} F_{\mathbf{k}^{\prime}}^{*}
\end{aligned}
$$

Here $N$ is the number of lattice points, $\Delta_{k}$ is the usual gap function which is assumed to be complex, and the asterisk denotes complex conjugation, while

$$
F_{\mathrm{k}}=\frac{\Delta_{\mathrm{k}}}{2 E_{\mathrm{k}}} \tanh \left(\frac{1}{2} \beta E_{\mathrm{k}}\right), \quad E_{\mathrm{k}}=\left(\varepsilon_{\mathrm{k}}^{2}+\Delta_{\mathrm{k}}^{2}\right)^{1 / 2} .
$$

$E_{\mathbf{k}}$ is the quasiparticle excitation energy, and $\beta=1 / k_{B} T$, where $k_{B}$ is Boltzmann's constant and $T$ denotes the absolute temperature. The gap parameter $\Delta_{k}$ searched for is the one minimizing the Gibbs energy at fixed chemical potential corresponding to a grand canonical system. Accordingly, we are looking for the stationary points of the Gibbs energy. Introducing $\Delta_{\mathbf{k}}=\delta_{\mathbf{k}} \exp \left(i \varphi_{\mathrm{k}}\right)$ and minimizing with respect to the modulus $\delta_{\mathrm{k}}$ and phase $\varphi_{\mathrm{k}}$ of the gap parameter, we get

$$
\begin{aligned}
& \delta_{\mathbf{k}}=\frac{1}{N} \sum_{\mathbf{k}^{\prime}} \cos \left(\varphi_{\mathbf{k}}-\varphi_{\mathbf{k}^{\prime}}\right) V\left(\mathbf{k}, \mathbf{k}^{\prime}\right) \delta_{\mathbf{k}^{\prime}} \frac{1}{2 E_{\mathbf{k}^{\prime}}} \tanh \left(\frac{1}{2} \beta E_{\mathbf{k}^{\prime}}\right) \\
& 0=\frac{1}{N} \sum_{\mathbf{k}^{\prime}} \sin \left(\varphi_{\mathbf{k}}-\varphi_{\mathbf{k}^{\prime}}\right) V\left(\mathbf{k}, \mathbf{k}^{\prime}\right) \delta_{\mathbf{k}^{\prime}} \frac{1}{2 E_{\mathbf{k}^{\prime}}} \tanh \left(\frac{1}{2} \beta E_{\mathbf{k}^{\prime}}\right)
\end{aligned}
$$

In Eqs. (7) we have split the equation $\partial G / \partial \delta_{k}=0$ into its real and imaginary parts. $\partial G / \partial \varphi_{\mathrm{k}}=0$ for all $\varphi_{\mathrm{k}}$ and $\delta_{\mathrm{k}}$ provided the pairing potential satisfies the symmetry relation $V\left(\mathbf{k}, \mathbf{k}^{\prime}\right)=V\left(\mathbf{k}^{\prime}, \mathbf{k}\right)$, which we shall assume in the fol- 
lowing. In this case $\varphi_{\mathbf{k}}$ is arbitrary, and from Eq. (7b) it follows that $\varphi_{\mathbf{k}}$ equals a constant $\varphi$ independent of the wave vector $\mathbf{k}$. Thus, in the general complex case, the gap parameter can be written in the form $\Delta_{\mathrm{k}}=\delta_{\mathrm{k}} \exp (i \varphi)$. Noting that the cos term in Eq. (7a) equals unity and multiplying by $\exp (i \varphi)$ in $(7 \mathrm{a})$, we get the usual BCS gap equation

$$
\Delta_{\mathbf{k}}=\frac{1}{N} \sum_{\mathbf{k}^{\prime}} V\left(\mathbf{k}, \mathbf{k}^{\prime}\right) F_{\mathbf{k}^{\prime}} .
$$

We note that the arbitrary phase $\varphi$ does not enter in the Gibbs energy, meaning that by varying $\varphi$ the Gibbs energy cannot be lowered further. Therefore, without loss of generality, we can restrict Eq. (8) to the case of a real gap parameter $\Delta_{k}$. Similarly, the bifurcation phenomena to be treated here will not be influenced by restricting the gap parameter to real values.

We shall concentrate on solutions of (8) corresponding to singlet pairing, $\Delta_{k}=\Delta_{-k^{\prime}}$. As a result of the choice (4) of the pairing potential, the solutions of the gap equation are guaranteed to be of the form

$$
\Delta_{\mathbf{k}}=2 \Delta_{x} \cos \left(k_{x} a_{x}\right)+2 \Delta_{y} \cos \left(k_{y} a_{y}\right) .
$$

Substitution of Eqs. (4), (6), and (9) into (8) gives a set of coupled nonlinear algebraic equations determining the gap parameters $\Delta_{x}$ and $\Delta_{y}$ :

$$
\begin{aligned}
& \Delta_{x}-\frac{1}{N} g_{x} \sum_{\mathbf{k}} \cos \left(k_{x} a_{x}\right) F_{\mathbf{k}}=f_{1}\left(\Delta_{x}, \Delta_{y}\right)=0, \\
& \Delta_{y}-\frac{1}{N} g_{y} \sum_{\mathbf{k}} \cos \left(k_{y} a_{y}\right) F_{\mathbf{k}}=f_{2}\left(\Delta_{x}, \Delta_{y}\right)=0 .
\end{aligned}
$$

The solutions of (10) are the stationary points of the Gibbs free energy, and if more than one stationary point exists, the physical relevant solution is the one with the lowest Gibbs energy. We note that if $\left(\Delta_{x}, \Delta_{y}\right)$ is a solution of (10), then so is $\left(-\Delta_{x},-\Delta_{y}\right)$. Furthermore, the solutions of the gap equation (10) are classified as follows: The cases $\Delta_{y}=\Delta_{x}$ and $\Delta_{x}=-\Delta_{y}$ are denoted $s$ - and $d$ wave pairings, respectively. All other cases where $\left|\Delta_{x}\right| \neq\left|\Delta_{y}\right|$ are denoted mixed $s$ - and $d$-wave pairings.

In solving the gap equation numerically, $A$ and $k_{B}$ are set equal to unity; this means that energies are measured in units of $A$, while temperatures are measured in units of $A / k_{B}$. Using the parameter value $A=0.05 \mathrm{eV}$ from Eq. (3), we obtain $A / k_{B}=580 \mathrm{~K}$. At a given $\mu$ and for a specific solution of (10), the filling $\rho$ of dopant holes in the band is determined by $^{23}$

$$
\rho=\frac{1}{2} \sum_{\mathbf{k}}\left(1-\frac{\varepsilon_{\mathbf{k}}}{E_{\mathbf{k}}} \tanh \left(\frac{1}{2} \beta E_{\mathbf{k}}\right)\right) \text {. }
$$

Here we interpret $\rho$ as the density of carrier holes relative to the Mott insulating phase. A relatively small value of $\rho$ then corresponds to a high density $x=1-\rho$ of electrons, leading to an approximately cylindrical Fermi surface, in agreement with angle-resolved photoemission experiments. ${ }^{7}$ For a given filling $\rho$, Eqs. (10) and (11) can be solved self-consistently for $\Delta_{x}, \Delta_{y}$, and $\mu$. From a physical point of view, this sounds appealing, as the filling $\rho$ (degree of doping) is expected to be known rather than the chemical potential $\mu$. However, from a mathematical point of view, this makes only sense when considering the physically correct solution with the lowest Gibbs energy. When studying bifurcation phenomena with several distinct solutions, it will only make sense to solve the gap equation (10) at a given $\mu$. This follows from the fact that $\Delta_{x}$ and $\Delta_{y}$ are the stationary points of the Gibbs free energy evaluated at fixed $\mu$, and hence the Gibbs energy can be directly compared for different solutions.

\section{BIFURCATIONS IN THE BCS GAP EQUATION}

Returning to the gap equation (10), we observe that, according to the implicit function theorem, the gap parameters $\Delta_{x}$ and $\Delta_{y}$ can be written as functions of $(T, \mu)$ in a neighborhood of a solution point, provided the Jacobian

$$
\underline{J}=\left[\begin{array}{ll}
\frac{\partial f_{1}}{\partial \Delta_{x}} & \frac{\partial f_{1}}{\partial \Delta_{y}} \\
\frac{\partial f_{2}}{\partial \Delta_{x}} & \frac{\partial f_{2}}{\partial \Delta_{y}}
\end{array}\right]
$$

taken at this solution point, is regular. As $T$ and $\mu$ are varied, the Jacobian $\underline{J}$ may become singular and a bifurcation may occur. Because of the symmetry properties of the BCS gap equation, the appearance of pitchfork bifurcations are expected as $T$ and $\mu$ are varied. In particular, by changing $T$, the transition from the normal state $\left(\Delta_{x}=\Delta_{y}=0\right)$ to the superconducting state $\left(\Delta_{x} \neq 0\right.$ and $\left.\Delta_{y} \neq 0\right)$ is a pitchfork bifurcation with a transition temperature denoted by $T_{c}$.

In order to identify strictly the pitchfork bifurcation at $T_{c}$, one can use the Liapunov-Schmidt reduction procedure to transform the two-dimensional gap equation (10) into a one-dimensional equation $h(x, T)=0$, which is equivalent to $x^{3}-T x=0$. An explicit expression for $h(x, T)$ cannot be found in the case of the BCS gap equation (10), but in principle arbitrarily close approximations to $h(x, T)$ can be computed numerically from the Liapunov-Schmidt procedure. We shall not go through the steps of this reduction procedure, because it is a little lengthy and for the identification of a pitchfork bifurcation we do not need $h(x, T)$ explicitly. What we need is a theorem from Ref. 24 stating that if $h$ satisfies

$$
h=h_{, x}=h_{, x x}=h_{, T}=0 \text { and } h_{, x x x} h_{, x T}>0,
$$

at a bifurcation point, the number of solutions of $h(x, T)=0$ jumps from one to three as $T$ crosses $T_{c}$ from above. The subscripts $x$ and $T$ succeeding the comma denote partial derivatives with respect to the state variable $x$ and bifurcation parameter $T$, respectively. Using results from 24 and 25, the partial derivatives of $h$ can be determined directly from $f_{1}$ and $f_{2}$ as follows. At $T=T_{c}$, where the solution $\left(\Delta_{x}, \Delta_{y}\right)=(0,0)$ bifurcates, one of the eigenvalues, say, $\lambda_{1}$, of the Jacobian (12) vanishes, while the other eigenvalue $\lambda_{2}$ is less than zero. Let $\underline{P}$ be the matrix diagonalizing the Jacobian $\underline{J}$ at 
$\left(\Delta_{x}, \Delta_{y}\right)=(0,0)$ and $T=T_{c}$, i.e,

$$
\underline{P}^{-1} \underline{J} \underline{P}=\left[\begin{array}{cc}
0 & 0 \\
0 & \lambda_{2}
\end{array}\right] \text {. }
$$

Instead of $\left(\Delta_{x}, \Delta_{y}\right)$, we introduce new gap variables $\left(w_{1}, w_{2}\right)$ according to

$$
\left(\begin{array}{l}
w_{1} \\
w_{2}
\end{array}\right)=\underline{P}^{-1}\left(\begin{array}{l}
\Delta_{1} \\
\Delta_{2}
\end{array}\right),
$$

and in terms of $\left(w_{1}, w_{2}\right)$, the gap equation (10) becomes

$$
\begin{aligned}
& 0=F_{1}\left(w_{1}, w_{2}, T\right)=f_{1}\left(\Delta_{x}, \Delta_{y}, T\right), \\
& 0=F_{2}\left(w_{1}, w_{2}, T\right)=f_{2}\left(\Delta_{x}, \Delta_{y}, T\right) .
\end{aligned}
$$

From 24 and 25 the partial derivatives of $h(x, T)$ at the bifurcation point $T=T_{c}$ and $x=0$ are determined by

$$
\begin{aligned}
& h_{, x x}=F_{1, w_{1} w_{1}}, \\
& h_{, x x x}=F_{1, w_{1} w_{1} w_{1}}-\frac{3}{\lambda_{2}} F_{1, w_{1} w_{2}} F_{2, w_{1} w_{1}}, \\
& h_{, T}=F_{1, T}, \\
& h_{, x T}=F_{1, w_{1} T}-\frac{1}{\lambda_{2}} F_{1, w_{1} w_{2}} F_{2, T},
\end{aligned}
$$

where the partial derivatives on the right-hand side are to be calculated at $\left(\Delta_{x}, \Delta_{y}\right)=(0,0)$ and $T=T_{c}$. By construction, $h$ and $h_{, x}$ are both 0 at the bifurcation point. Performing numerical differentiation of Eqs. (17) using the parameter values in Eq. (3), $g_{x}=g_{y}=3.0$ and $\mu=-2.3$, the following values of the derivatives in Eq. (13) have been found at the bifurcation point $T=T_{c}=0.541: \quad h_{, x x}=-6.6 \times 10^{-12}, \quad h_{, T}=-1.5$ $\times 10^{-19}, h_{, x x x}=3.60$, and $h_{, x T}=0.81$, showing that within numerical errors Eq. (13) is fulfilled and accordingly the bifurcation at $T_{c}$ is a pitchfork bifurcation of the above-mentioned type. The relevance of showing that we have a pitchfork bifurcation is connected to perturbations of the bifurcation diagram. The qualitative picture of the perturbed pitchfork bifurcation is known and simple, whereas a more degenerate bifurcation gives a more complicated bifurcation diagram in the perturbed case, even though this additional degeneracy in the unperturbed case possesses the same bifurcation diagram as the pitchfork bifurcation.

\section{NUMERICAL RESULTS}

In this section we shall investigate multiple solutions of the gap equation (10) and their properties as the parameters $\mu$ and $T$ are varied. In particular, we shall be interested in pitchfork bifurcations and perturbations of the pitchfork bifurcations due to asymmetric pairing interaction in the $x y$ plane. The influence of the nonlinear properties of the gap equation on the transition temperature and superconducting density of states will be discussed.

The gap equation solves for the stationary points of the Gibbs energy, and in order to get global information about these solution points as parameters are varied, contour plots of the Gibbs energy prove useful. Figure 2 shows two contour plots of the Gibbs energy at $\mu=-2.0$ and $T=0$ using the tight-binding parameters in Eq. (3). Figure 2(a) displays a situation with symmetric pairing interaction $g_{x}=g_{y}=1.20$, and Fig. 2(b) shows a situation with asymmetric pairing interaction $g_{x}=1.20$ and $g_{y}=1.18$. In physical units, $g_{x}=1.20$ corresponds to $g_{x}=1.2 A=0.06 \mathrm{eV}$. Similar values of the pairing strength, ranging from 0.043 to $0.065 \mathrm{eV}$, have been used in an analogous model in Ref. 11 dealing with a spinpolaron pairing mechanism. The tight-binding spectrum applied in Ref. 11 corresponds to Eq. (2) with $B=C=0$ and $A=0.0896 \mathrm{eV}$. Our choice of parameter values is then consistent with the spin-polaron pairing mechanism suggested in Ref. 11. In Fig. 2(a) four local minima, four saddle points, and a local maximum at $\left(\Delta_{x}, \Delta_{y}\right)=(0,0)$ are present. The local minima are all stable degenerate physical solutions. The saddle points and local maximum are unstable equilibrium points. In Fig. 2(b) the asymmetric case $\left(g_{x}=1.20\right.$ and $\left.g_{y}=1.18\right)$ is shown. The main result of the symmetry breaking is to increase the depth of two of the four local minima, leaving two stable physical solutions with lower energy. The contour plots provide a quick overview of the possible solutions, their stability, and physical relevance with respect to the requirement of lowest energy. Furthermore, initial guesses for numerical procedures used to solve the gap equation are

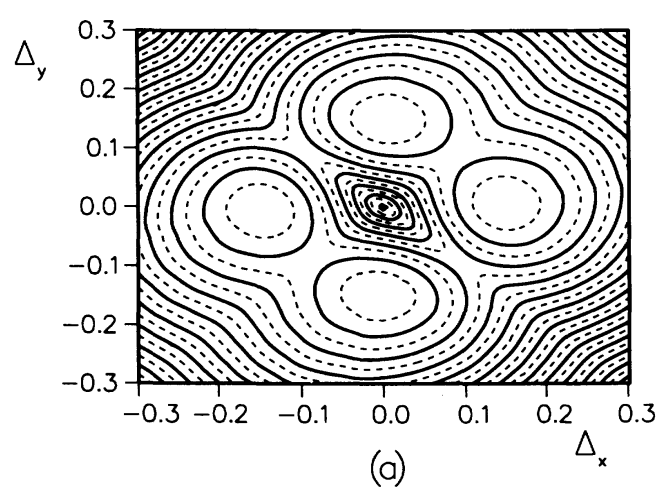

(a)

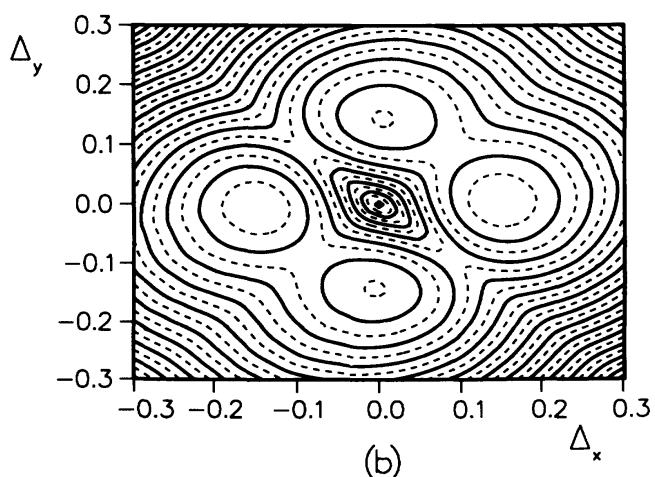

FIG. 2. Contour plots of the Gibbs free energy at $\mu=-2.0$ and $T=0$. (a) Symmetric case $g_{x}=g_{y}=1.20$. (b) Asymmetric case $g_{x}=1.20$ and $g_{y}=1.18$. 
easily found from contour plots of the Gibbs energy.

In Fig. 3(a) we follow all the equilibrium points as the chemical potential is varied in the case of symmetric pairing interaction with $g_{x}=g_{y}=1.20$. Both $\Delta_{x}$ and $\Delta_{y}$ are shown as function of $\mu$. Solid lines denote local stable points, and dashed lines are unstable ones. As $\mu$ is increased from -2.5 to 0.0 , we observe first a pitchfork bifurcation at $\mu=-2.45$ separating the normal state from a $s$-wave superconducting state. At $\mu=-2.25$ two unstable saddle points with $d$-wave symmetry emerge from a pitchfork bifurcation. The next pitchfork bifurcation happens at $\mu=-2.12$, where a transition from $s$-wave pairing to a mixed $s$ - and $d$-wave pairing takes place. This is an example of a second-order phase transition with a nonzero order parameter both above and below the transition point. ${ }^{24,26}$ The mixed $s$ - and $d$-wave solution turns into a pure $d$-wave solution at $\mu=-1.80$, also as a result of a pitchfork bifurcation. Just before $\mu=0$, the gap gradually vanishes.

The results from Fig. 3 resemble the Landau theory of phase transitions for systems with two order parameters and with free energy equal to a fourth-order polynomial in the order parameters. ${ }^{26}$ In this polynomial only terms of even degree are present. Accordingly, we can view the bifurcation diagrams in Fig. 3 more easily by following the equilibrium points directly in the $\left(\Delta_{x}, \Delta_{y}\right)$ plane. For $\mu$ less than the first bifurcation point at $\mu=-2.45$, $\left(\Delta_{x}, \Delta_{y}\right)=(0,0)$ is a global minimum of the Gibbs energy. After the bifurcation the center point $(0,0)$ turns into a saddle and two minima are created ( $s$-wave solutions),

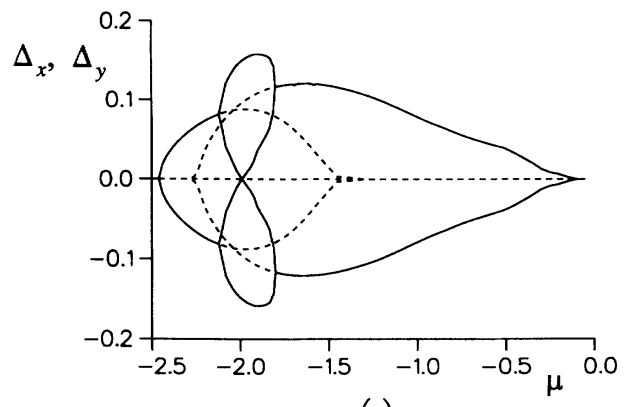

(a)

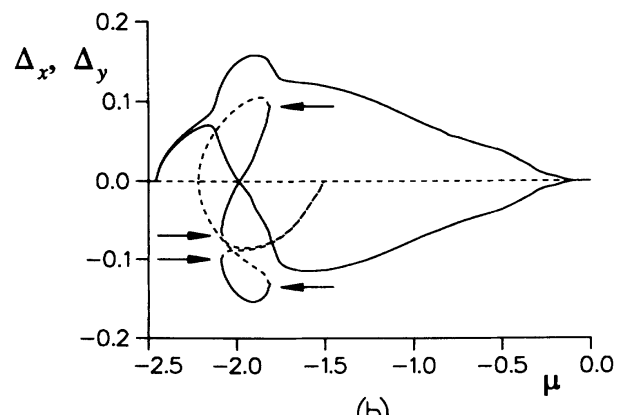

(b)

FIG. 3. Bifurcation diagram showing $\Delta_{x}$ and $\Delta_{y}$ vs $\mu$ at $T=0$. (a) Symmetric case $g_{x}=g_{y}=1.20$. (b) Asymmetric case $g_{x}=1.20$ and $g_{y}=1.18$. The arrows mark the saddle-node bifurcations. which move away from the center along the line $\Delta_{x}=\Delta_{y}$. At $\mu=-2.25$ the center saddle bifurcates into a local maximum and two saddles are created moving along the line $\Delta_{x}=-\Delta_{y}$ ( $d$-wave symmetry). At $\mu=-2.12$ the two local minima turn into saddle points and around each of these new saddles two local minima are created [see also Fig. 2(a)]. The two saddles move toward the center as $\mu$ is increased and collide with the center at $\mu=-1.45$. The four local minima move from the first and third quadrants into the second and fourth quadrants (the mixed $s$ - and $d$-wave solutions) and collide with the saddles in the second and fourth quadrants, creating two minima (the pure $d$-wave solutions). The minima defining the $d$-wave solutions move toward the center and collide with the center just before $\mu=0$.

Figure 3(b) displays the gap parameters $\Delta_{x}$ and $\Delta_{y}$ as function of $\mu$ in the asymmetric case with $g_{x}=1.20$ and $g_{y}=1.18$. For clarity, not all fixed points are shown. The points which are not displayed are found by a symmetry operation about the line $\Delta_{x}=0$. The figure resembles Fig. 3(a) except for the perturbation of the two pitchfork bifurcations separating the transitions between the $s$-wave, mixed $s$ - and $d$-wave, and $d$-wave solutions. These two transitions become smooth or continuous for the asymmetric pairing interaction as we follow the two local minima in Fig. 2(b) corresponding to the lowest Gibbs energy. The other two local minima, with slightly higher Gibbs energy and with the two saddles close by each of them, emerge from a saddle-node bifurcation. ${ }^{24,25}$ The saddle-node bifurcations are marked by arrows in Fig. 3(b). The result of the asymmetric pairing interaction is to go from a sharp second-order phase transition to a smooth change from one phase to the other.

In Fig. 4 we show the transition temperature $T_{c}$ versus the filling $\rho$ of dopant holes for the symmetric case (solid line) and asymmetric case (dashed line). Because of the very small difference between the coupling strength in the $y$ direction in the two cases, only a very small difference in $T_{c}$ is observed. The qualitative behavior of $T_{c}$ versus $\rho$ is in agreement with experimental results ${ }^{4}$ except from the fact that superconductivity in our model sets in already at $\rho=0$. Comparing experimental data of $T_{c}$ versus hole concentration with our calculated $T_{c}$ versus $\rho$, an offset of $\rho \approx 0.07$ should be added to compensate for

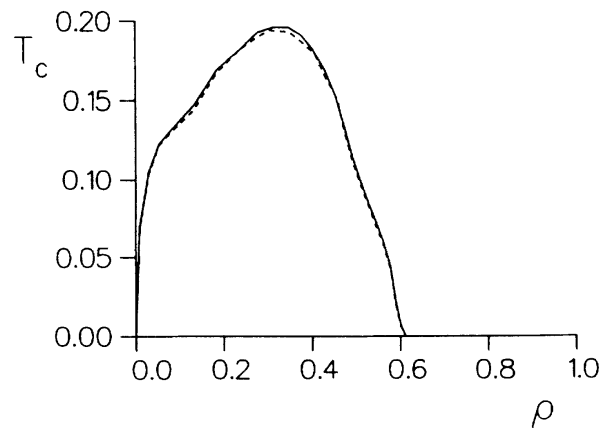

FIG. 4. Critical temperature $T_{c}$ vs $\rho$ for the symmetric case $g_{x}=g_{y}=1.20$ (solid curve) and for the asymmetric case $g_{x}=1.20$ and $g_{y}=1.18$ (dashed curve). 
the initial Mott insulating phase. The model in Eq. (1) does not contain Coulomb repulsive terms, and accordingly the model is metallic for all densities $\rho$. The fact that $T_{c} \rightarrow 0$ as $\rho \rightarrow 0$ in Fig. 4 is a pure mathematical property of the solutions of Eq. (10). Around $\rho=0.35$ the transition temperature attains its maximum value $T_{c}=0.2$, corresponding to a physical transition temperature of $116 \mathrm{~K}$, which is a representative value for hightemperature superconductors.

Figure 5 depicts the density of states in the symmetric case for $\mu=-0.1,-1.0,-2.0$, and -2.3 corresponding to the normal $d$-wave, mixed $s$ - and $d$-wave, and $s$-wave states, respectively. The density of states for the asymmetric case deviates infinitely little from the symmetric case and is therefore not shown. Blowups of the regions around $\varepsilon_{\mathrm{k}}=0$ are shown in Fig. 6. The $d$-wave and mixed $s$ - and $d$-wave states show no gap, whereas the $s$-wave state at $\mu=-2.3$ shows a gap of this size $0.3 A=15$ $\mathrm{meV}$. This value is somewhat lower than the value $\Delta=24$ $\mathrm{meV}$ obtained from the photoemission measurements reported in Ref. 27. However, closer to the pitchfork bifurcation separating the $s$-wave and mixed $s$ - and $d$-wave phases, we expect a larger gap.

Figure 7 shows the bifurcation diagram for the symmetric pairing interaction at $\mu=-1.9$ by varying the temperature $T$. The solid lines are the stable fixed points, and the dashed lines denote saddle points. Figure 7 clearly reveals two pitchfork bifurcations separating the normal, $d$-wave, and mixed $s$ - and $d$-wave states.

In the asymmetric case with $g_{x}=1.20$ and $g_{y}=1.18$, the pitchfork bifurcation separating the mixed $s$ - and $d$ wave phase from the pure $d$-wave phase disappears (Fig. 8). The transition between the two phases becomes smooth, and in addition a saddle-node bifurcation emerges close to the previous pitchfork bifurcation. Note that the pitchfork bifurcation at the transition tempera-
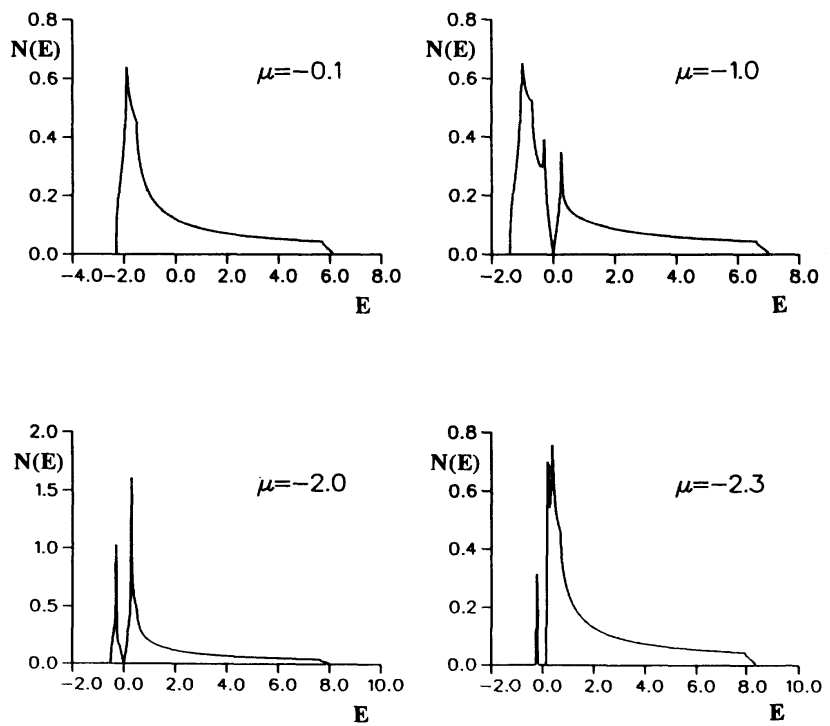

FIG. 5. Superconducting density of states for the symmetric case $g_{x}=g_{y}=1.20$ at $T=0$ for $\mu=-0.1$ (normal state), $\mu=-1.0$ ( $d$-wave solution), $\mu=-2.0$ (mixed $s$ - and $d$-wave solution), and $\mu=-2.3$ (s-wave solution).
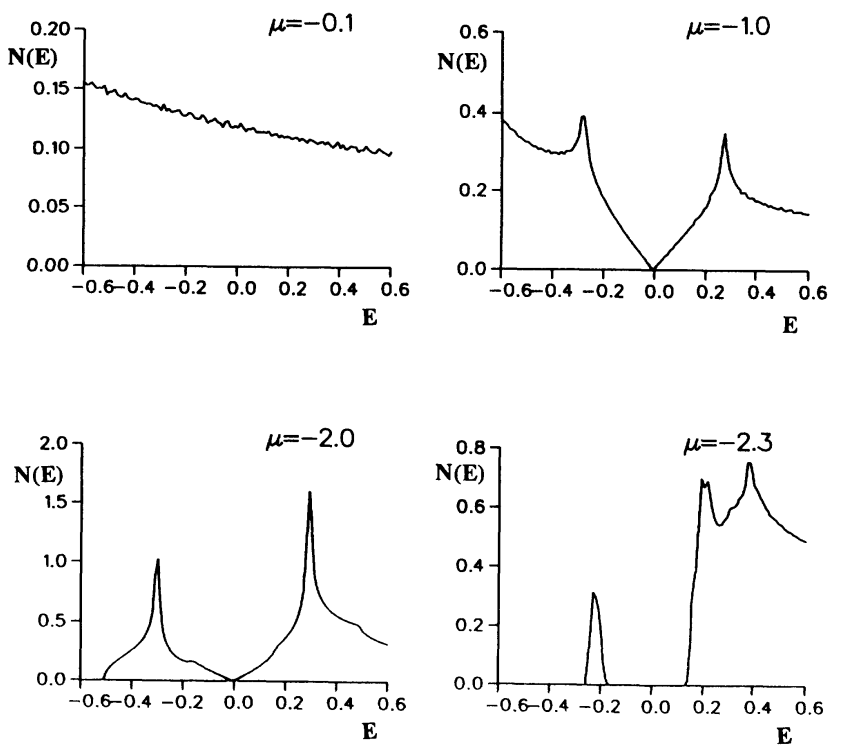

FIG. 6. Blowup of the regions around the Fermi energy in Fig. 4.

ture $T_{c}$ between the normal and superconducting states remains a pitchfork bifurcation in the case of asymmetric pairing interaction.

The abrupt change in the gap parameters in the symmetric case and the smooth change of the gap parameters in the asymmetric case will have a dramatic effect on measurable quantities such as the electronic specific heat ${ }^{23}$

$$
C=T \frac{d S}{d T}=\frac{1}{2} k_{B} \beta^{2} \sum_{\mathbf{k}}\left[E_{\mathbf{k}}+\beta \frac{d E_{\mathbf{k}}}{d \beta}\right] E_{\mathbf{k}} \operatorname{sech}^{2}\left(\frac{1}{2} \beta E_{\mathbf{k}}\right)
$$

and the spin susceptibility ${ }^{23}$

$$
\chi=\frac{1}{8} \mu^{2} \beta \sum_{\mathbf{k}} \operatorname{sech}^{2}\left(\frac{1}{2} \beta E_{\mathbf{k}}\right) .
$$

$S$ denotes the entropy. This is illustrated in Figs. 9 and 10 , showing $C$ versus $T$ and $\chi$ versus $T$, respectively, for $\mu=-1.9$. The solid lines depict the results with symmetric pairing interaction, and the dashed lines show the results with asymmetric pairing interaction. When the

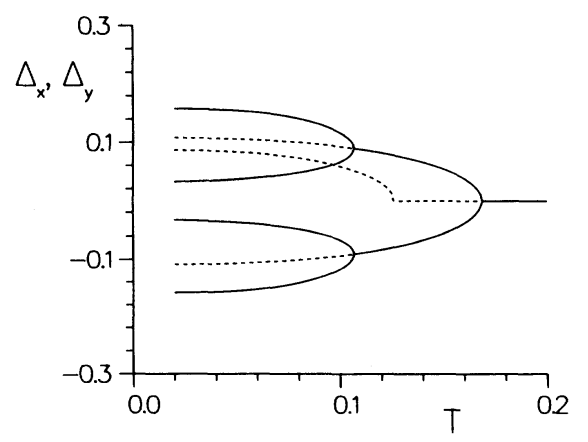

FIG. 7. Bifurcation diagram showing $\Delta_{x}$ and $\Delta_{y}$ vs the temperature $T$ at $\mu=-1.9$ in the symmetric case $g_{x}=g_{y}=1.20$. 


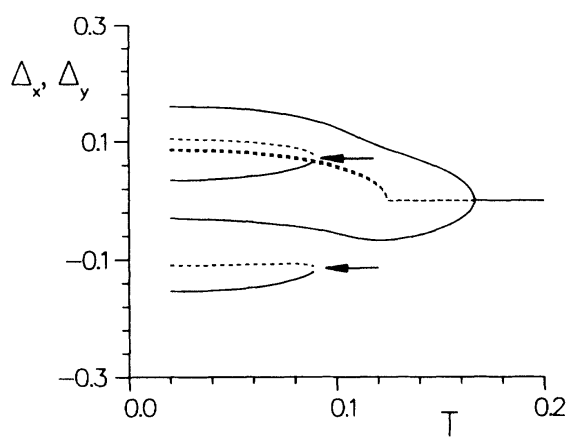

FIG. 8. Bifurcation diagram showing $\Delta_{x}$ and $\Delta_{y}$ vs the temperature $T$ at $\mu=-1.9$ in the asymmetric case $g_{x}=1.20$ and $g_{y}=1.18$. The arrows mark the saddle-node bifurcations.

gap parameter changes abruptly as a result of a pitchfork bifurcation, the specific heat makes a jump and the derivative with respect to $T$ of the spin susceptibility makes a jump. ${ }^{28}$ When the gap parameter varies smoothly from one phase to another, $C$ and $\chi$ also vary smoothly.

Reference 29 provides a review of recent experimental measurements of the specific heat versus temperature for high- $T_{c}$ superconductors. Some experimental samples reveal two well-resolved peaks, whereas certain other samples show only one peak. In the context of the BCS model, this fact can be related to whether the sample show a transition from mixed $s$ - and $d$-wave superconductivity to pure $s$ - or $d$-wave superconductivity, or the chemical potential has a value allowing only a pure $s$ - or $d$-wave symmetry in the BCS gap equation. Furthermore, Ref. 29 develops a generalized Ginzburg Landau theory with two order parameters to describe successfully the two peaks appearing in the specific heat of certain samples of high$T_{c}$ materials. The phase transitions in Ginzburg-Landau theory are evident from contour plots of the free-energy functional. These contour plots show the same phenomenological features as the Gibbs free energy from BCS theory. Accordingly, the Ginzburg-Landau description is in fully accord with the BCS model, also in the case of two order parameters.

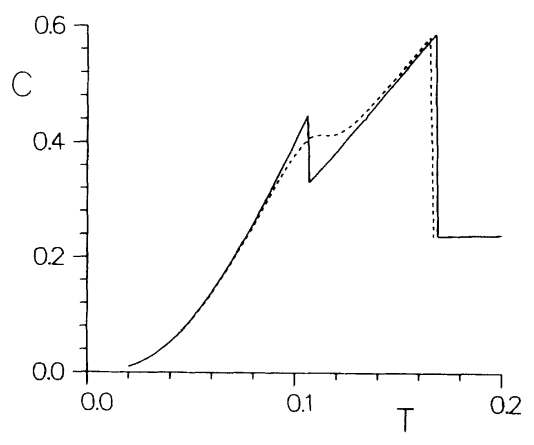

FIG. 9. Electronic specific heat $C$ vs $T$ at $\mu=-1.9$. (a) Symmetric case $g_{x}=g_{y}=1.20$ (solid curve). (b) Asymmetric case $g_{x}=1.20$ and $g_{y}=1.18$ (dashed curve).

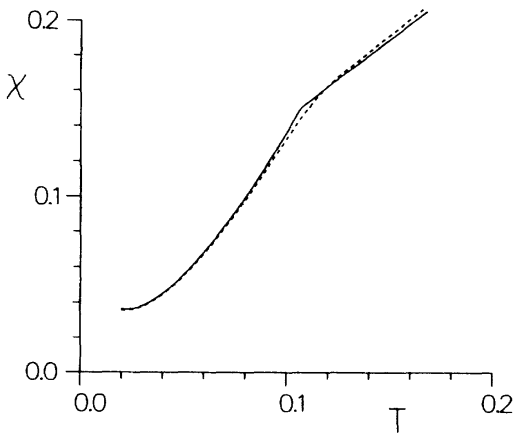

FIG. 10. Spin susceptibility $\chi$ vs $T$ at $\mu=-1.9$. (a) Symmetric case $g_{x}=g_{y}=1.20$ (solid curve). (b) Asymmetric case $g_{x}=1.20$ and $g_{y}=1.18$ (dashed curve).

Finally, we wish to comment on the possibility of having an on-site interaction term in the pairing potential $V\left(\mathbf{k}, \mathbf{k}^{\prime}\right)$ in Eq. (4). Preliminary calculations show that on-site attractive interaction favors $s$-wave pairing and repulsive on-site interaction favors $d$-wave pairing. For weak on-site repulsive interaction as well as for weak attractive interaction, mixing occurs between simple $s$-wave states with $\Delta_{\mathrm{k}}=\Delta_{0}=$ constant and extended states $\left(\Delta_{x} \neq 0\right.$ and $\Delta_{y} \neq 0$ ). As the on-site pairing parameter is varied, bifurcations take place as well. As detailed study of the role of the on-site pairing interaction is currently in progress.

\section{CONCLUSION}

The nonlinear properties of the BCS gap equation have been investigated in the case of anisotropic nearestneighbor pairing interaction in the planes of a high- $T_{c}$ superconductor using a tight-binding description of the holes. The dependence of the gap-state variables on the auxiliary parameters $T$ and $\mu$ has been mapped out, and the solutions of the gap equation have been classified according to $s$-wave, $d$-wave, and mixed $s$ - and $d$-wave symmetries.

In the symmetric case with identical pairing interaction in the $x$ and $y$ directions, pitchfork bifurcations separate the normal, $s$-wave, $d$-wave, and mixed $s$ - and $d$ wave superconducting phases as the bifurcation parameters $T$ and $\mu$ vary.

In the asymmetric case with slightly different pairing interaction in the $x$ and $y$ directions, the pitchfork bifurcations separating the $s$-wave, mixed $s$ - and $d$-wave, and $d$-wave superconducting phases are perturbed, and saddle-node bifurcations replace the original pitchfork bifurcations, leading to smooth transitions between the pure $s$ - and $d$-wave states and the mixed state. The pitchfork bifurcation separating the normal state from the superconducting state is not affected by the above symmetry breaking.

The electronic specific heat and spin susceptibility reveal singularities close to a bifurcation point. A pitchfork bifurcation manifests itself as a discontinuity in the specific heat and as a discontinuity in the slope of the 
spin susceptibility. Perturbing the pitchfork bifurcation removes these discontinuities.

The appearance of two singularities in the specific heat is in accordance with recent experimental facts. As the chemical potential is varied, the singularity resulting from a transition from one superconducting state to another may disappear. This could explain why some high- $T_{c}$ superconductors show only one peak in the specific heat, while others show two peaks. Finally, the qualitative features of the BCS Gibbs free energy exactly resembles the features of the Ginzburg-Landau freeenergy functional depending on two order parameters.

\section{ACKNOWLEDGMENTS}

This work is supported by the Danish National Science Research Council (Grant No. 11-7075) and the EC Science programme [Contract No. SCI/0229-C(AM)]. From one of us (MPS) special thanks goes to T. Schneider for the encouragement to study the nonlinear properties of the BCS gap equation and for helpful comments. P. Gross is acknowledged for indispensable help concerning the Liapunov-Schmidt procedure. We would also like to thank P. L. Christiansen, A. C. Scott, and T. Pavlopoulos for valuable discussions.
${ }^{1}$ V. J. Emery, Phys. Rev. Lett. 58, 2794 (1987).

${ }^{2}$ V. J. Emery and G. Reiter, Phys. Rev. B 38, 4547 (1988).

${ }^{3}$ M. Jarrell, H. R. Krishnamurthy, and D. L. Cox, Phys. Rev. B 38, 4584 (1988).

${ }^{4}$ J. E. Hirsch and F. Marsiglio, Phys. Lett. A 140, 122 (1989).

${ }^{5}$ T. Schneider, H. De Raedt, and M. Frick, Z. Phys. B 76, 3 (1989).

${ }^{6}$ T. Schneider and M. P. Soerensen, Z. Phys. B 80, 331 (1990).

${ }^{7}$ T. Schneider and M. P. Soerensen, Z. Phys. B 81, 3 (1990).

${ }^{8}$ R. Micnas, J. Ranninger, and S. Robaszkiewicz, Rev. Mod. Phys. 62, 113 (1990).

${ }^{9}$ J. E. Hirsch, Phys. Lett. A 138, 83 (1989).

${ }^{10} \mathrm{~K}$. Tanaka, Y. Yamaguchi, and T. Yamabe, Physica C 116, 53 (1990).

${ }^{11}$ H. Kamimura, S. Matsuno, and R. Saito, Solid State Commun. 67, 363 (1988).

${ }^{12}$ R. F. Wood, M. Mostoller, and J. F. Cooke, Physica C 165, 97 (1990).

${ }^{13}$ M. Rona, Phys. Rev. B 42, 4183 (1990).

${ }^{14}$ A. S. Davydov, Phys. Status Solidi B 146, 619 (1988).

${ }^{15}$ E. Kolley and W. Kolley, Phys. Lett. A 149, 233 (1990).

${ }^{16} \mathrm{M}$. P. Soerensen and T. Schneider (unpublished).

${ }^{17}$ R. Pankaluoto and K. Kaski, Phys. Scr. T 33, 227 (1990).

${ }^{18}$ H. De Raedt, T. Schneider, and M. P. Soerensen, Z. Phys. B
79, 327 (1990).

${ }^{19}$ J. F. Annett and R. M. Martin, Phys. Rev. B 42, 3929 (1990).

${ }^{20}$ M. S. Hybertsen, M. Schluter, and N. E. Christensen, Phys. Rev. B 39, 9028 (1989).

${ }^{21}$ F. Minami, T. Kimura, and S. Takekawa, Phys. Rev. B 39, 4788 (1989).

${ }^{22}$ T. Takahashi, H. Matsuyama, H. Katayama-Yoshida, Y. Okabe, S. Hosoya, K. Seki, H. Fujimoto, M. Sato, and H. Inokuchi, Phys. Rev. B 39, 6636 (1989); Nature 334, 691 (1988).

${ }^{23}$ A. J. Leggett, Rev. Mod. Phys. 47, 331 (1975).

${ }^{24}$ M. Golubitsky and D. G. Schaeffer, Singularities and Groups in Bifurcation Theory (Springer-Verlag, New York, 1985).

${ }^{25}$ P. Gross, M.Sc. thesis, Mathematical Institute, The Technical University of Denmark, DK-2800 Lyngby, Denmark 1990.

${ }^{26}$ E. L. Nagaev and A. I. Podel'shchikov, Phys. Lett. A 144, 473 (1990).

${ }^{27}$ C. G. Olson, R. Liu, A.-B. Yang, D. W. Lynch, A. J. Arko, R. S. List, B. W. Veal, Y. C. Chang, P. Z. Jiang, and A. P. Paulikas, Science 245, 731 (1989).

${ }^{28}$ H. Mori, J. Phys. Soc. Jpn. 58, 1394 (1989).

${ }^{29}$ T. C. Choy, M. P. Das, and Hongxing He, Phase Transit. 20, 1 (1990). 